\title{
Simposio: Evaluación de la Calidad Calidad de la Atención
}

\author{
DRA. BEATRİZ ZURITA GARZA \\ Master en Economia Aplicada, Ph. D. en Ciencias Epidemiológicas. \\ Cordinadora de Analisis de Potiticas de Salud en FUNSALUD
}

La calidad de atención es algo que se debe definir claramentc. para que se pueda medir, la calidad de la atcnción liene varias propiedades:

1. Consecuencias para la Salud

2. Cosios

3. Aceptabilidad del Usuario

4. Accptabilidad Social

\section{CONSECUENCIAS PARA LA SALUD}

Se refiere a la eficacia, efectividad.

La Eficacia es la habilidad de mejorar el eslado de salud a traves de la atención médica. Y la Efectividad es la habilidad de lograr el máximo beneficio de salud a través de la mejor atención médica.

\section{costos}

La eficioncia de los coslos es la habilidad de disminuir los cosios sin disminuir los beneficios de salud y la oplimidad, que es el mejor balance entre los costos y beneficios.

\section{ACEPTABILIDAD DEL USUARIO}

Es la participación, conveniencia, conforl.

\section{ACEPTABILIDAD SOCIAL}

Es la equidad dol acceso y los beneficios.

\section{CONCEPTO DE CALIDAD}

Incluye el nivel ideal de salud que implica disminuir el espacio que scpara el estado de salud actual con el estado optimo csperado. Implica un desarrollo iecnológico de la ciencia.

La calidad es la diferencia cntre lo alcanzable y lo alcanzado.

\section{DIMENSIONES DE LA CALIDAD EN EL ENCUENTRO PACIENTE - PROVEEDOR}

(Momento de la verdad)

El proveedor puede ser una enfermera, médico u olro. Se liene que hacer lo siguiente:

- Mancjo del proccso de la atcución a la salud.

- Mancio del problema de salud presenado.

- Mancjo preventivo.

- Relación Intcrpersonal.

- Condiciones Ambientales

(Lomado de A. Dunabedian)
Todo esto liene que ver con las dimensiones de la aceptabilidad es decir:

La accesibilidad, relación médico - paciente, amcnidades, preferencias de los pacientes; todo esto en dos aspecios:

- Sobre los efectos de la atención

- Sobre los costos de la atención

Si no se logra que el paciente acepte la interacción no se logrará brindar una alención adecuada.

La accesibilid.ed liene que ver con el tiempo de espera hasta recibir la atención. se observa que en el sector privado la espera es menor que en el Ministerio de Salud y en el Seguro Social.

\section{DIMENSIONES DE LA ATENCION AL USUARIO}

Al usuario lo que más le interesa es:

1 Sus valores, preferencias y necesidades

2. Coordinación, integración y hujo de información porque son individuos.

3. Tener información, comunicación y educación

4. Comodidad física

5. Apoyo emocional y el control del miedo y la ansiedad.

6. La participación de la fimilia y los amigos

7. La transición y continuidad do la alención.

Dentro del respeto a los valores, preferencias y necesidades de los pacientes, estos toman en cuenta:

- Calidad de vida

- Participación en la toma alc decisiones

- Dignidad

- Neccsidades y autonomía

En términos de coorúmación, integración y' flujo dc información, es necesario saber que a los pacientes les interesa que cxista:

- Coordinación c integración de la atención de prímera linca a los pacientes. Es importante la primcra persona que tienc contaclo con cl paciente.

- Coordinación c integración de los servicios auxiliares y de apoyo.

- Coordinación e integración de la alenciún médica.

Que de la información. comunicación y cóucación, el paciente requiere distintas informaciones tales como:

- Información del eslado clínico, de los avances udel pronósico

- Información sobre el proceso de la afención

- Inlormacion y Educación para faciliar la autonumía, autocuidado y la promoción de la salued. 
Comodidad física, tener en cuenta que el paciente necesila de ayuda con las actividades diarias, manejo del dolor y conocimientos del ambiente del sitio de la alención.

El apoyo emocional y apoyo para el control del miedo y la ansledad, están relacionadas con la nccesidad de alivio de la ansiedad sobre el estado clínico, el tratamiento y el pronóstico. Asi mismo con la necesidad de alivio de la ansiedad sobre el impacto de la enfermedad, sobre el individuo, la familia y con el alivio de la ansiedad sobre el impacto financiero de la enfermedad.

La participación de la familia y los amigos, implica que el equipo de salud identifique los lugares que ocupan los familiares y amigos del paciente, la participación de la familia en la toma de decisiones, el apoyo. La familia como proveedora de atención y el reconocimiento de las necesidades de la familia.

Hay que respetar la autonomía del paciente.

Es muy importante la transición y continuidad, que abarca el alta del paciente y los chequeos siguientes donde es importante la información, coordinación, planeación del cuidado y el apoyo respeclivo.

Hay que ayudar al paciente durante el proceso de alta, ¿cómo se irá, con quién etc.?

El promedio de tiempo de duración por consulta médica es de 20 a 30 minutos, lo que amerita preguntar ¿todo esto se puede realizar en este promedio de tiempo?

\section{MODELO PARA LA EVALUACION DE LA CALIDAD DE LOS SERVICIOS DE SALUD}

Disponibilidad de recursos productividad

Capacidad de producir servicios

Accesibilidad

Utilización de Servicios

Tecnologia de la Salud

Satisfacción de Necesidades (calidad de la atención)

(A. Donabedian)

Las dimensiones de la calidad son tres:

- Técrica

- Interpersonal y

- Comodidades

Pero viendo lo que generalmente se conoce se tiene: Estructura-Proceso y Resultado.

La ESTRUCTURA, con todos los atributos materiales y organizacionales de los sitios en los que se proporcionan la atención, es la base para una buena atención relativamente estable; algunos indicadores son fáciles de medir, sin embargo tienen baja correlación con los medios de proceso y resultado. Son indicadores de estruclura :
El número, tipo y calificación de personal, los lipos de servicio disponibles y la distancia de la población usuaria.

EI PROCESO, es todo lo que los médicos y otros proveedores de atención hacen por los pacientes, incluyendo la habilidad con la que llevan a cabo la atención.

Tienen que ver indicadores tales como:

El número de visitas por médico, por unidad de tiempo, el número de exámenes de laboratorio por paciente, por diagnósti. co entre otros.

Evalúa el proceso, es importante porque se pueden deteciar deficiencias específicas, asi como los responsables.

El RESULTADO, es lo que se obtiene para el paciente en términos de un cambio en el estado de salud - para bien o para mal - que puede atribuirse a la atención bajo evaluación. Concierne lo que es deseable o posible de lograr con una buena atención, evalúa el cumplimiento del objetivo de la atención y es muy necesario poderle atribuir a un proceso para su evaluación.

Son sus indicadores: El conocimiento sobre la enfermedad, cambio en las conductas para la salud y la satisfacción del paciente y del proveedor.

EVALUACION DEL SISTEMA DE SALUD

Dimensión ESTRUCTURA PROCESO RESULTADO

Enfoque

Por lo tanto se puede hacer una evaluación de la estructura tomando en cuenta que si se tiene suficientes recursos y el diseño organizacional es apropiado, se puede proteger y promover la calidad.

La evaluación de la calidad de atención a través del proceso que es el objeto primario de la evaluación, se puede hacer a través de la observación directa y de la información registrada, esto lleva un tiempo pero en realidad si se quiere cambiar las cosas, se tiene que evaluar los procesos.

¿Qué indicadores debemos tener?

- Utilidades en el plano nacional.

- Patrón que permite comparar el propio progreso (en el tiempo).

- Poner de manifiesto los logros a lin de corregir el desequilibrio y eliminar las disparidades. 


\title{
Evaluación de la Calidad Indicadores
}

\author{
DRA. FARIA BERNUI BOBADILLA
}

Oficina de Garantia de Calidad HNERM - IPSS

\section{INDICADORES - CONCEPTO}

"Son variables que sirven para medir los cambios".

INDICADORES: Principales errores de concepto

* Confundir al indicador como meta.

* No analizar los procesos involucrados en el fenómeno evaluado por el indicador.

* No evaluar las dispersiones del promedio planteado.

* Diseñar y formular indicadores estratégicos sin cabal conocimiento y comprensión de los procesos de la operación por evaluar.

* No considerar el resultado del indicador como herramienta de gestión para la toma de las decisiones.

\section{INDICADORES}

Dificultades en su implantación

* Información oportuna para el diseño

* Entendimiento y aceptación por parte de los directivos.

* Compromiso de las unidades gestión.

* Registros adecuados y oportunos.

* Estandarización de los procesos.

* Sistema de informática inflexibles.

\section{INDICADORES}

¿Cómo se Implantan?

* Decisión y compromiso de los directivos.

* Participación en los diseños del personal operativo.

* Selección de indicadores estratégicos.

* Difusión y capacitación.

* Compromiso en su aplicación.

* Retroalimentación y corrección.

* Análisis de los procesos de las desviaciones.

\section{INDICADORES}

Cualidades

* Disponibilidad: información fácil de conseguir.

* Simplicidad: de interpretación sencilla.

* Calidad: Registros veraces.

* Objetividad: Los resultados invarialbles.

* Validez: Expresa lo que intenta medir.

* Especificidad: refleja cambios exclusivos del fenómeno.

* Sensibilidad: Capta los cambios ocurridos.

\section{INDICADORES}

Utilidad

* Permiten determinar metas de salud, planificar los recursos y servicios necesarios.

* Instrumentos que facilitan la evaluación de resultados y la administración eficiente de las prestaciones.

* Ayudan a evaluar las dispersiones o varianzas anormales, para la oportuna toma de decisiones.

* Herramientas que nos permite desarrollar el proceso de mejoramiento continuo de la calidad.

* Permiten realizar comparaciones y tendencias de cambio en el tiempo.

* Trazadores que demuestren el avance en el logro de ob. jetivos y metas.

\section{INDICADORES}

Clasificación Sistemica

* Estructura.

* Proceso.

* Resultado.

Indicador de Concentración de Consultas 1984 - 1995

\section{$\mathrm{N}^{\circ}$ de Consultas Externas $/ \mathrm{N}^{\circ}$ de Consultantes}

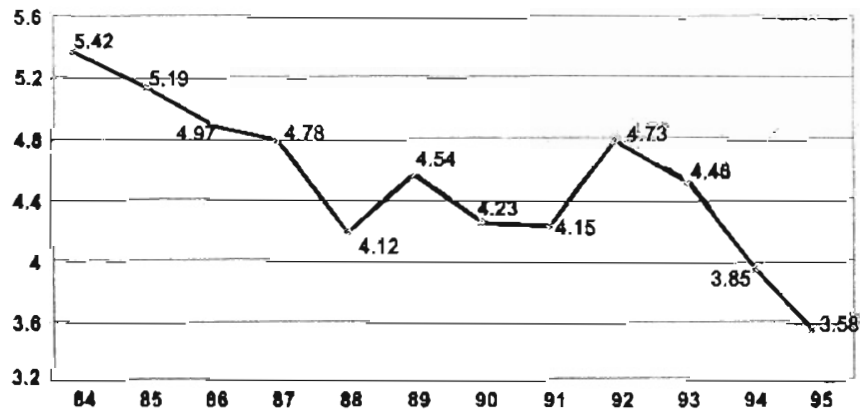

Concentración de consultas Tasa promedlo: $2.77 \%$ anual de crecimiento Concentración: es un indicador anual el estándar en el IPSS es 4 (valor Nacional). 
Indicador de Recetas por Consultantes 1990 - 1995

$N^{\circ}$ de Recetas Despachadas de Consultantes $/ N^{\circ}$ de consultantes

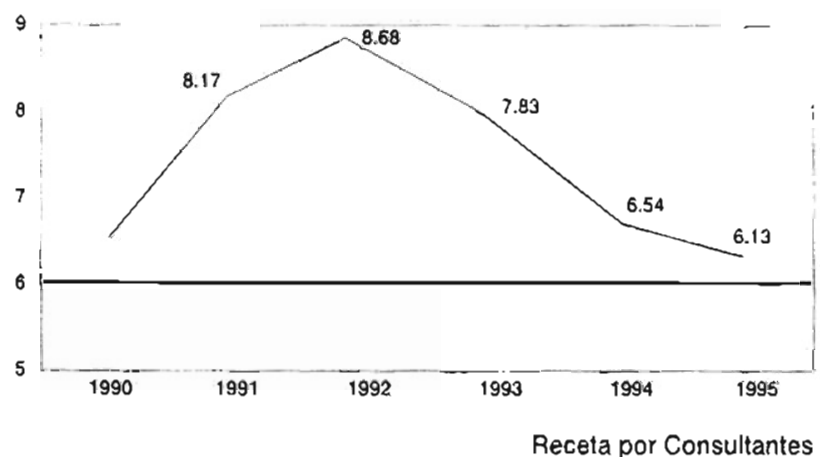

Es un indicador anual. Está relacionado a la concentración. Debe anallzarse en conjunto con olras vatiables. En este cuadro, es el año 1990 el resultado se acerca al estandar por que existia carencia de formación.

Indicador de Promedio de Permanencia

$1984-1995$

\section{Estancias / Egresos}

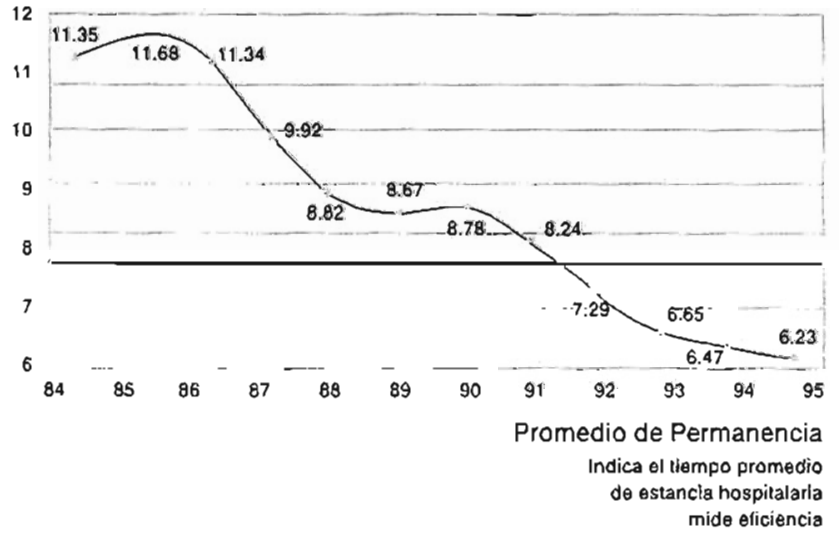

Indicador de Porcentaje de Ocupación

$1984-1995$

(Pacientes Dia / Días Cama) x100

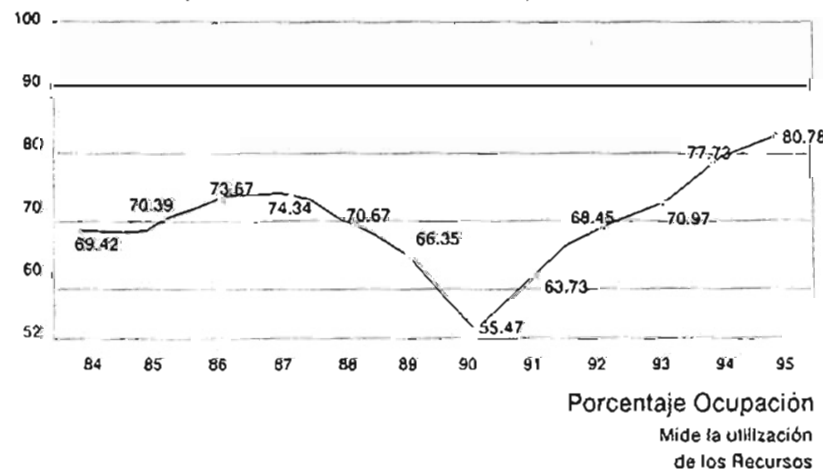

Evolución tiempa de espera
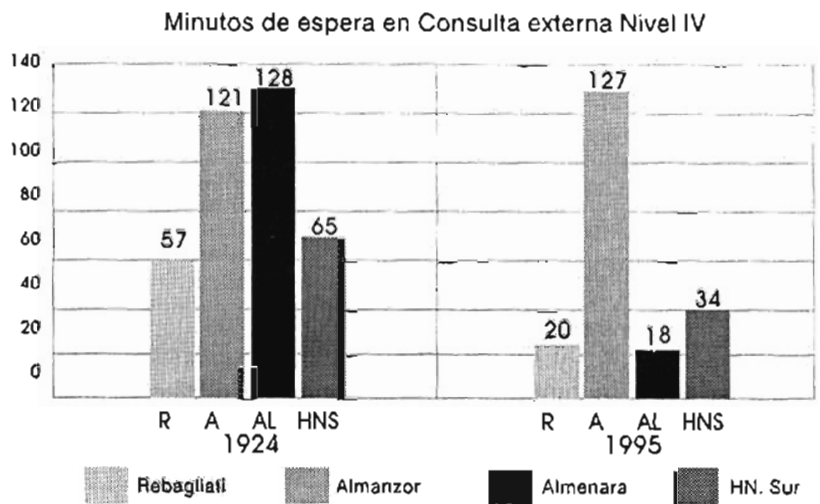

Indicador de calidad $\alpha \theta$ Consulta Externa: Mide el tiempo desde que un paciente Ingresa al hospital hasta que se le olorga una clta.

Indicador de Promedio de Permanencia $1990-1995$

Estancias / Egresos

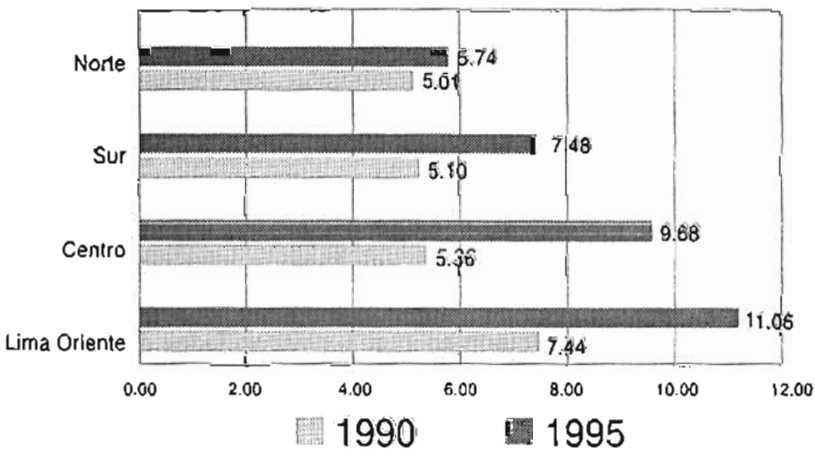

Compara el promedio de tiempo de estancia entre el año 1990 y 1995 por macro región IPSS

Indicador de Intervalo de Sustitución $1990-1995$

(Días Cama Disponible - Pacientes)/Egresos

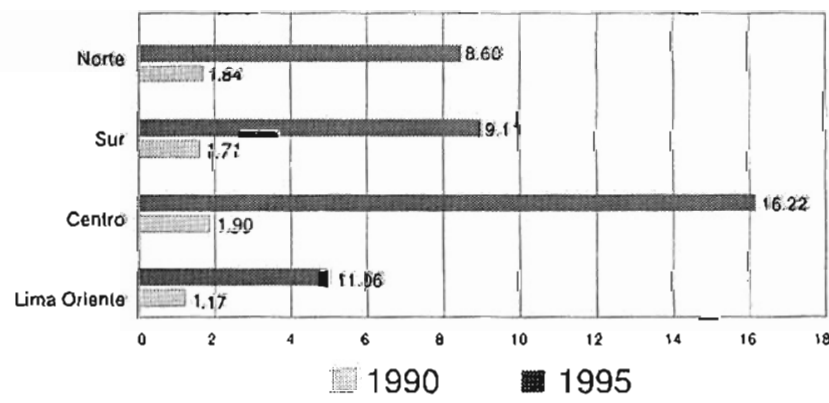

Mide los dias que una cama de servicro de inlernamiento, queda libre o no es ullizada El intevalos de sustlueión no debe ser mayor que 1. 